\title{
The chemical content of nearby galaxies: NGC 147
}

\author{
D. R. Gonçalves ${ }^{1}$, L. Magrini ${ }^{2}$ P. Leisy ${ }^{3}$ \& R. L. M. Corradi ${ }^{3,4}$ \\ ${ }^{1}$ IAG - Universidade de São Paulo, Brazil; Email: denise@astro.iag.usp.br \\ ${ }^{2}$ INAF, Osservatorio di Arcetri, Italy; ${ }^{3}$ Instituto de Astrofísica de Canarias, Spain; ${ }^{4}$ Isaac \\ Newton Group of Telescopes, Spain
}

\begin{abstract}
The dwarf spheroidal galaxy NGC 147 is one of the companions of the Andromeda galaxy. In this contribution we show the first spectroscopic results for the PN population of NGC 147, obtained from the GEMINI Multi-Object Spectrograph (GMOS) in December 2005. We present our results on the physical and chemical properties of the planetary nebulae. At variance with the behaviour of the old stellar population of the galaxy, the metallicity of $\mathrm{PNe}$ is roughly constant through the galaxy. Moreover, if the \pm 0.06 dex of the $[\mathrm{Fe} / \mathrm{H}]$ to $[\mathrm{O} / \mathrm{H}]$ conversion is considered, there is no difference between the RGB and the PNe metallicities. We also show that the PNe in NGC 147 are 1 to 10 Gyr-old. Therefore, these results suggest that NGC 147 had no significant enrichment for a long period of time.
\end{abstract}

Keywords. Galaxies: abundances - Local Group - individual (NGC 147)

\section{The Local Group dwarf spheroidals}

Are dwarf ellipticals and spheroidals the evolved descendant of previous star-forming dwarfs? A way of answering this question is through the study of the metallicity-luminosity relation of the dwarf galaxies - the LG has plenty of them-determined from PNe, which are present in both early- and late-type galaxies.

NGC 147 is one of the three (NGC 205, NGC 185 and NGC 147) dwarf companions of M 31. Contrary to its sister galaxy -NGC 185- NGC 147 is gas and dust free (Mateo 1998). These two galaxies have very similar star formation histories and are dominated by the old stellar population (Mateo 1998). On the other hand they have different PNe populations, since PNe in NGC 185 are systematically brighter than those in NGC 147 (Corradi et al. 2005). The stellar metallicity of the galaxy was studied by Han et al. (1997) and Nowotny et al. (2003). These authors pointed out that the NGC 147 star formation has ceased at least 1 Gyr ago, since it has no MS stars with $M_{V}<1$, and that its younger stars are more centrally concentrated than the majority of its older stars.

\section{The chemistry of NGC 147}

The spectroscopic data were obtained with the GMOS at GEMINI North, in December 2005. We observed 8 PNe in the mask, which had long-slits 1 arcsec wide and, combining the two configurations, ranging approximately from $370 \mathrm{~nm}$ to $1000 \mathrm{~nm}$. Our chemical analysis allow us to derive elemental abundances of $\mathrm{He}, \mathrm{O}, \mathrm{N}, \mathrm{Ne}, \mathrm{Ar}, \mathrm{S}$. The following analysis of the chemical evolution of this galaxy is based on the oxygen abundances, which, in a first approximation, do not vary during the stellar lifetimes, thus representing the chemistry of the galaxy at the time the PN progenitors were born. In this study we obtain abundances empirically, using the ICF (ionization correction factors) method, as 
well as from the photoionization modeling (CLOUDY) of each PN. Abundances were derived using the observed auroral lines to determine $\mathrm{T}_{e}[\mathrm{O}$ III $]$ and/or $\mathrm{T}_{e}[\mathrm{~N}$ II $]$, allowing an accurate determination of the ionic and total abundances.
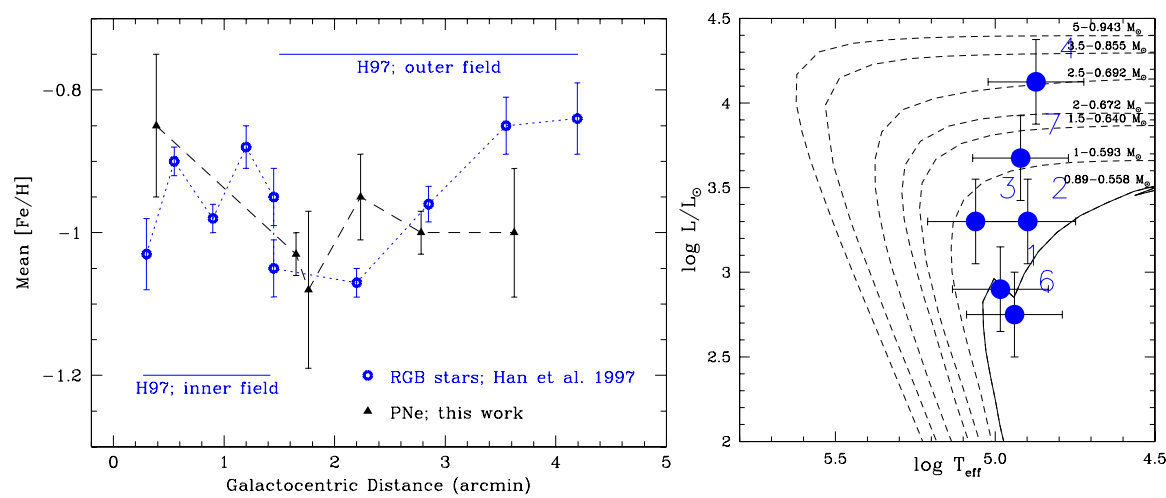

Figure 1. Left:The RGB and PN metallicities of NGC 147. $[\mathrm{Fe} / \mathrm{H}]_{\mathrm{PNe}}=[\mathrm{O} / \mathrm{H}]_{\mathrm{CLOUDY}}-0.37$; with $[\mathrm{O} / \mathrm{H}]_{\text {solar }}=8.66$ (Asplund 2003). The average outer field corresponds to $[\mathrm{Fe} / \mathrm{H}]=-1.00$, while for the inner field $[\mathrm{Fe} / \mathrm{H}]=-0.91$. Right: The NGC 147 PNe evolutionary tracks. These are the H- and He-burning tracks, for $\mathrm{Z}=0.004$, from Vassiliadis \& Wood (1994).

We show in Fig.1 the comparison between the metallicity given by the old population RGB stars and that of the PN population. Han et al. (1997) have interpreted their results as indicating a weak evidence for increasing metallicities with the galactocentric distance -note the mean values they found for the inner and the outer regions of NGC 147 . This work, on the other hand, shows that, within the errors, the PN metallicity is constant throughout the galaxy. If we also consider the $\pm 0.06 \mathrm{dex}$ of the $[\mathrm{Fe} / \mathrm{H}]$ to $[\mathrm{O} / \mathrm{H}]$ conversion (Mateo 1998), there is no difference between the RGB and the PN metallicities. The central star parameters plotted in the right part of Fig. 1 were obtained from our CLOUDY modeling of the various $\mathrm{PNe}$ of the galaxy, having as constraints the observed flux at [O III] $5007 \AA$ and the HeI/HeII line ratio. The evolutionary tracks give us the stellar masses and therefore the progenitor masses. From them we are able to discuss the chemical history of NGC 147, using the age-metallicity relation for the PNe in the galaxy. We note from Fig.1 that the PNe in NGC 147 are 1 to 10 Gyr-old and that all of them have similar metallicities.

All the above arguments allow us to state that there was no significant enrichment in NGC 147, for a long period of time.

\section{Acknowledgements}

DRG would like to thank the partial support of FAPESP (03/09692-0, 04/11837-0) and the organizers for an IAU grant.

\section{References}

Asplund, M. 2003, CNO in the Universe, ed. C. Charbonnel, D. Schaerer, \& G. Meynet (San Francisco: ASP), ASP Conf. Ser., 304, 275

Corradi R. M. L. et al. 2005, A\&̈A 438, 437

Han M., Hoessel J. G., Gallagher J. S., Holtsman J., \& Stetson P. B. 1997, AJ 113, 1001

Mateo M. L. 1998A, ARAA 36, 435

Nowotny W., Kerschbaum F., Olofsson H., \& Schwarz H. E. 2003, A $\& A$ 403, 93

Vassiliadis E., \& Wood P. R. 1994, ApJS 92, 125 